\title{
Cell Block as a Tool of Diagnostic Refinement in Cytological Examination of Pleural and Peritoneal Effusions
}

\author{
Dr. Aswini Gude $\mathrm{MD}^{1}$, Dr. Danda Mani Mala MD ${ }^{2 *}$, Dr. Varun Kamidi MD \\ ${ }^{1}$ Assistant Professor, Department of Pathology, Gayatri Institute of Health Care \&Medical Technology, GVP Medical College, Maridi Valley, \\ Marikavalasa, Visakhapatnam -Andhra Pradesh PIN-530048, India \\ ${ }^{2}$ Assistant Professor, Department of Pathology, Gayatri Institute of Health Care \&Medical Technology, GVP Medical College, Maridi Valley, \\ Marikavalasa, Visakhapatnam -Andhra Pradesh 530048, India \\ ${ }^{3}$ Assistant Professor, Department of Pathology, Gayatri Institute of Health Care \&Medical Technology, GVP Medical College, Maridi Valley, \\ Marikavalasa, Visakhapatnam -Andhra Pradesh 530048, India
}

DOI: $\underline{10.36348 / \text { sjpm.2020.v05i10.004 }}$

| Received: 09.10.2020 | Accepted: 23.10.2020 | Published: 29.10.2020

*Corresponding author: Dr. Danda Mani Mala MD

\section{Abstract}

Cytological examination of body fluids enables us to examine and differentiate between inflammatory, benign and malignant effusions. Cell block (CB) technique is method of cytological examination of serous fluids besides conventional smear (CS) technique. The aim of this study was to compare the role of cell block technique over the conventional smear technique in the cytological diagnosis of serous fluids. This was a prospective study done at our medical college and tertiary care hospital between 2017 to 2020 . A total of 114 patients were included in the study that underwent paracentesis for effusion cytology. Equal quantities of pleural and peritoneal fluid received and prepared into CS and CB for examination. Cellularity, architecture patterns, morphological features and yield for malignancy were compared, using the two methods. 71 were pleural fluid and 43 were peritoneal fluids. CS and CB comparison for cellularity showed statistically significant $(\mathrm{p}<0.05)$ and comparison of malignant effusion done with McNemar's test for CB showed that CS method was not superior to CB method in pleural, peritoneal and total body effusions. The stastistical analysis of malignant effusions $(n=114)$ for cell block method for sensitivity was $100 \%$, specificity $79.63 \%$, positive predictive value $21.43 \%$ and negative predictive value $100 \%$. The CB method provides high cellularity, better architectural patterns, morphological features and an additional yield of malignant cells than CS method. CB method is superior to CS method.

Keywords: Conventional smear, Cell Block, Serous effusion, Peritoneal effusion, Mesothelial cells, Malignant effusions. Copyright () 2020 The Author(s): This is an open-access article distributed under the terms of the Creative Commons Attribution 4.0 International License (CC BY-NC 4.0) which permits unrestricted use, distribution, and reproduction in any medium for non-commercial use provided the original author and source are credited.

\section{INTRODUCTION}

Fluid accumulation in serous cavities in excess of the normal amount is referred as effusion. Fluid specimens are invaluable specimens and help in diagnosis of particular lesional pathology, identifying the immediate precipitating factors causing the effusion and results in specific diagnosis. Cytological evaluation is the best way to detect the presence of malignancy in body cavity fluids. The general cytological examination can be performed easily, quickly, and inexpensively. Although a positive diagnosis is highly reliable, a negative result does not rule out malignant causes[1]

Cytological examination of serous fluids is one of the commonly performed investigations. It is important not only in the diagnosis of malignant lesions, but also helps in staging and prognosis. The information regarding various non-infectious and infectious conditions like bacterial, viral fungal and parasitic infections of serous membranes can also be assessed[2].

Accurately diagnosing cells as either malignant or reactive mesothelial cells in serous effusions is a common diagnostic problem. The lower sensitivity of cytodiagnosis of effusions is mainly attributable to overcrowding or overlapping of cells, cell loss, and changes due to different laboratory processing methods[2]. The serous effusion is the representative of much larger surface area than that obtained by needle biopsy.

Reactive mesothelial cells, abundance of inflammatory cells, and paucity of representative cells contribute to considerable difficulties in making conclusive diagnosis in conventional smear[3]. 
Cell block (CB) method is another method of cytological diagnosis for serous effusions. Cell block method is one of the oldest methods, for the evaluation of body cavity fluids[2]. Due to cellular overlapping, delaying artefact, suboptimal processing, preparative cytotechnique and leaving behind useful material causes lower diagnostic yield in conventional smear (CS) method. The residual material can be very useful in increasing diagnostic yield by cell block method. Cell block method increases the sensitivity of detecting malignancies, and also has the ability to reduce false positive interpretations[4].

Hence the present study was undertaken to emphasize the role of cell block technique over conventional smear in serous effusions.

\section{MATERIAL AND METHODS}

The present prospective study was carried on 114 fluid specimens from patients who underwent paracentesis for the effusion cytology by CS \& CB method in the cytology section, Dept of Pathology, at our tertiary care medical college and hospital between 2017 to 2020.

Fresh samples of pleural and peritoneal fluids received in cytology section were first examined with the naked eye. In case of haemorrhagic fluid $0.1 \%$ glacial acetic acid was added to haemolyse red blood cells. $10 \mathrm{ml}$ fresh fluid sample was taken and divided into two equal parts $(5 \mathrm{ml}$ each) for conventional cytology and cell block technique.

Conventional Smear technique: For conventional smear $5 \mathrm{ml}$ of fluid was centrifuged at $1000 \mathrm{rpm}$ for 5 minutes and a minimum of two thin smears were prepared from the sediment. One smear was immediately fixed with $95 \%$ alcohol and stained with Haematoxylin-Eosin (H\&E) and other was stained with Leishman stain.

Cellblock technique: $5 \mathrm{ml}$ of fluid was immediately fixed in $10 \%$ alcohol-formalin in 1:1 proportion for one hour and centrifuged at 1000rpm for $5 \mathrm{~min}$. The supernatant was discarded and $3 \mathrm{ml}$ fresh $10 \%$ alcohol-formalin was added to the sediment and was kept for minimum of 24hours. The sediment scooped out on the filter paper was processed like routine histopathological specimen. Paraffin embedded 4-6 $\mu$ thick sections were stained with HaematoxylinEosin stain. Special stains like Periodic Acid Schiff (PAS), Alcian Blue and Mucicarmine were performed wherever necessary.

Interpretation of conventional smear versus cellblock: In conjunction with clinical data the smears were categorized as benign, suspicious and malignant lesions following morphological criteria such as cellularity, arrangement (acini, papillae and cell balls), cytoplasmic and nuclear details as described by Khan et al[5]. Comparative evaluation of conventional smear versus cellblock preparations was done and cytomorphological characters were studied to identify the malignancy and most probable primary site. Statistical analysis was done by chi-square test.

\section{RESULTS}

Total of 114 body cavity fluid samples subjected to smear and cell block techniques, out of which 71(62\%) were pleural fluids and 43 (38\%) peritoneal fluids with male preponderance among the former and female predominance among latter. The maximum number of total samples in the present study was in the $5^{\text {th }}$ decade. The maximum number of samples was in $4^{\text {th }}$ decade in male and $5^{\text {th }}$ decade in female. Least number of samples was in the $2^{\text {nd }}$ decade.

Both in pleural and peritoneal fluids cellularity was more in cell block method as compared to conventional smear method. Architectural patterns, like cell balls, papillae, glands and three dimensional clusters were better appreciated in cell block than in conventional smears. Both in pleural and peritoneal fluid samples sheets, glandular pattern and papillary structures were more commonly observed in cell block as compared to conventional smears whereas singly scattered cells were predominantly seen in conventional smear.

Pleural Fluid Analysis: Out of the 71 pleural fluid samples cytological diagnosis of benign effusion was rendered in $58(82 \%)$ cases and suspicious for malignancy in $11(15 \%)$ cases and 02 cases as malignant. 56 cases were diagnosed as benign and 15 $(21 \%)$ cases as malignant effusions in cell block method. There was no diagnosis of suspicious for malignancy in cellblock.

Out of 71 cases, discrepancy was noted in 17 cases. Analysis of these 17 cases of pleural fluid samples showed that 06 cases which were reported as benign effusions in conventional smear, were diagnosed as malignant lesions by cell block method. Out of these 6 cases, 03 cases were reported as benign due to singly scattered cells. 02 cases were misdiagnosed, as morphology was obscured by hemorrhagic background, plenty of inflammatory cells, necrotic material and reactive mesothelial cells. 01 case was reported as inflammatory smear due to predominant neutrophils, lymphocytes, macrophages and few RBCs. Out of the 11 cases which were reported as suspicious for malignancy, 07 cases were diagnosed as malignant effusions. By cell block method, additional 13 cases were detected as malignant i.e. $18 \%$ more diagnostic yield for malignancy.

Peritoneal fluid analysis: Out of 43 peritoneal fluid samples, cytological diagnosis of malignant effusion was rendered in 4 cases $(10 \%)$ and 7 cases were diagnosed as suspicious for malignancy. 
Remaining 32 cases were diagnosed as benign effusions. By cellblock method 13 cases $(30 \%)$ were diagnosed as malignant effusions and remaining 30 cases as benign effusions.

04 benign cases of conventional smears were diagnosed as malignant lesions by cell block method. In 03 cases, morphological features were obscured by hemorrhagic background, necrotic material inflammatory cells and reactive mesothelial cells. 01 case was reported as florid mesothelial hyperplasia due to wide range of reactive changes. Out of 07 cases diagnosed as suspicious for malignancy in conventional smear method, 05 were diagnosed as malignant and another 02 as benign lesions by cell block method. Cell block method had $21 \%$ more diagnostic yield for malignancy.

Total number of 28 body fluid samples was diagnosed as malignant effusions by cellblock method which included 15 pleural and 13 peritoneal fluids. In all 28 cases diagnosis of metastatic malignant effusion was rendered.

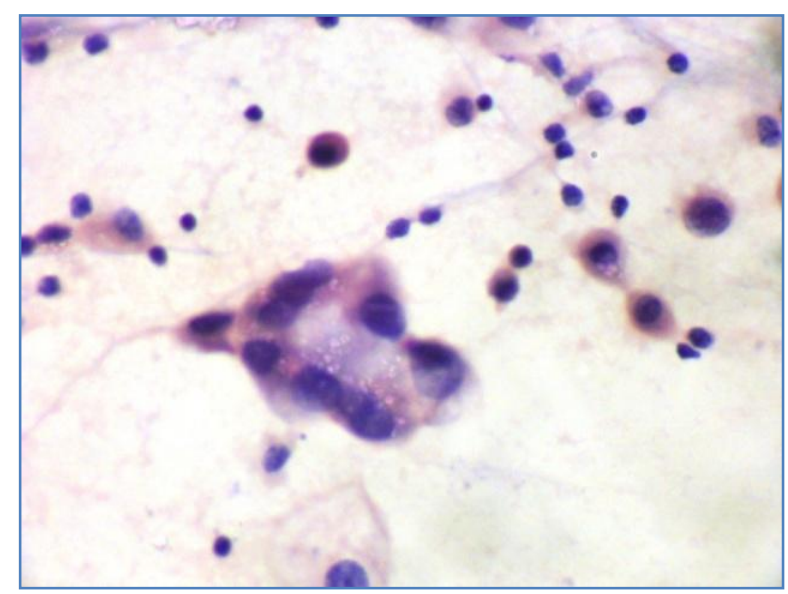

Fig-1(a): CS- Mucinous adenocarcinoma

H\&E (40X) Malignant cells forming a gland and having intra cytoplasmic mucin vacuoles

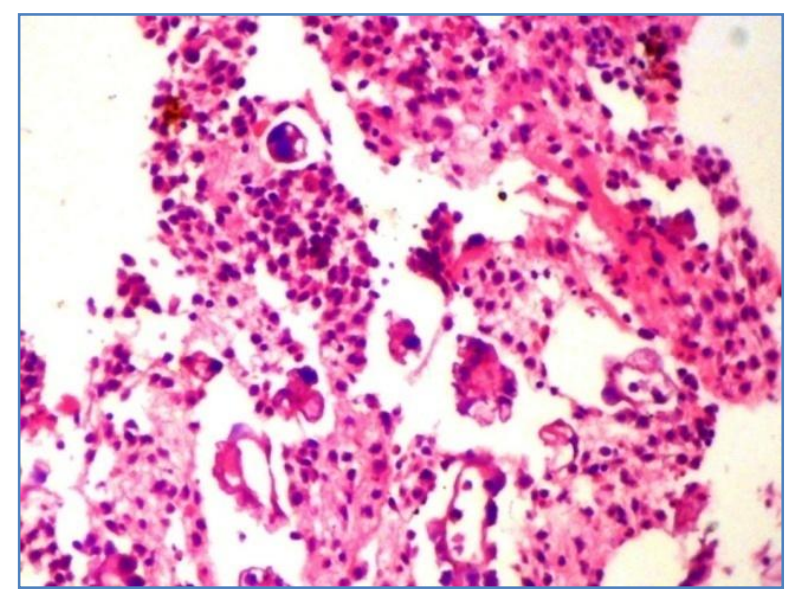

Fig-1(b): CB-Mucinous adenocarcinoma

H\&E (10X) Malignant cells in glandular pattern with few signet ring cells

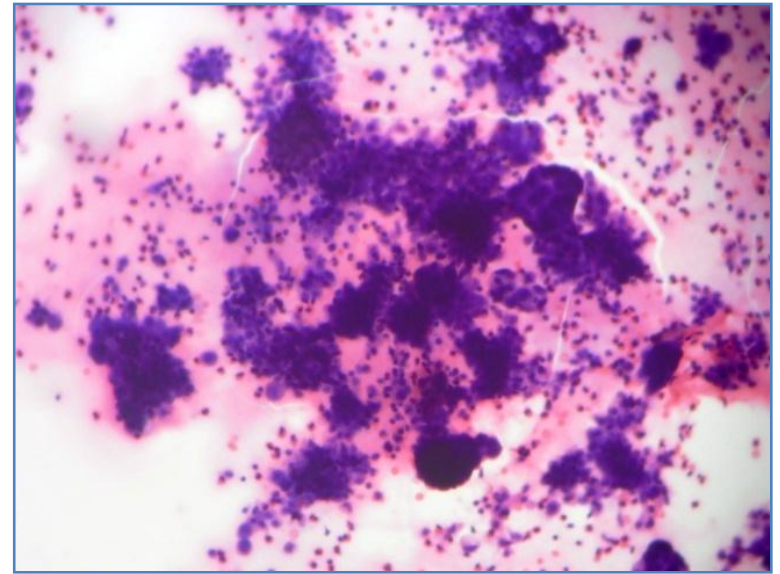

Fig-2(a): CS-Adenocarcinoma in plueral fluid

H\&E (10X) markedly cellular smear showing malignant cells in clusters and balls.

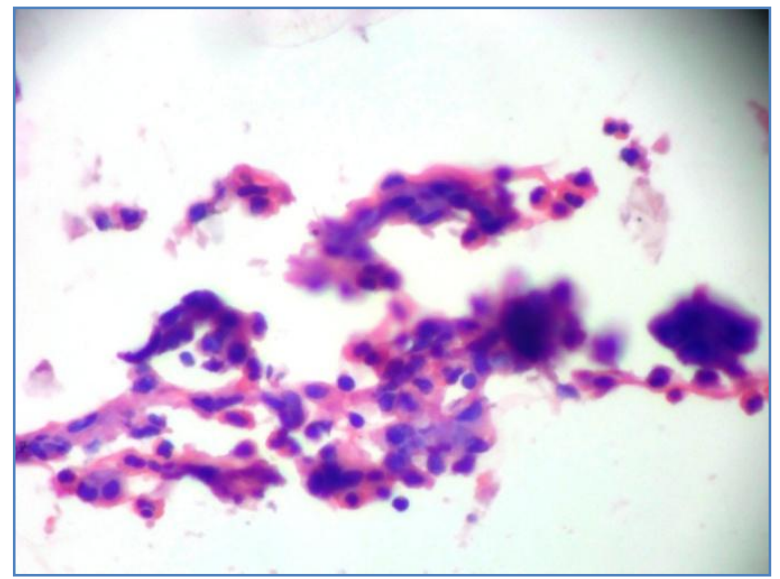

Fig-2(b): CB- Glandular pattern (Adenocarcinoma)

H\&E(40X) Malignant cells forming glandular structures.

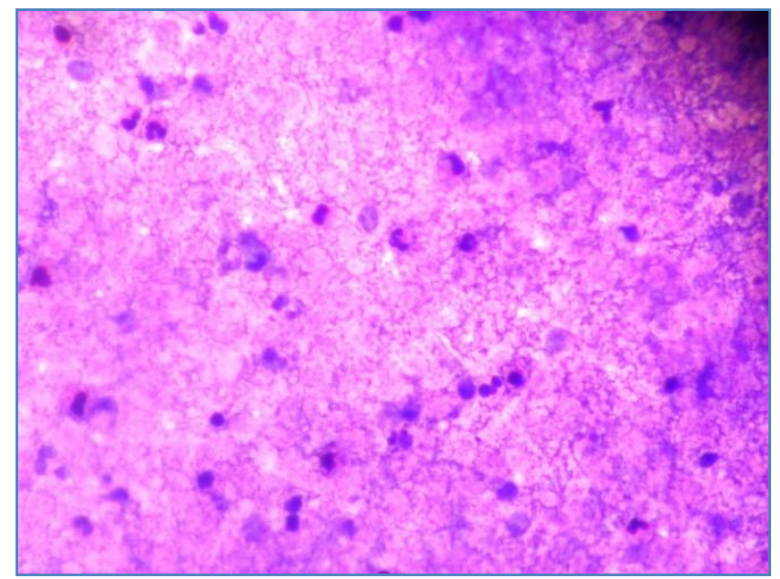

Fig-3(a): CS- Tuberculosis

H\&E (40X) Abundant necrosis with lymphocytes and few epitheloid cells. 


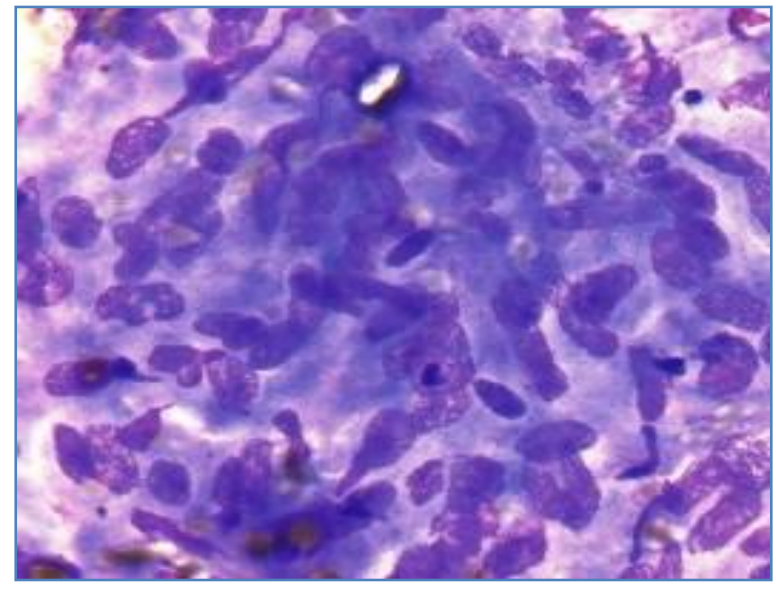

Fig-3(b): CB- Tuberculosis

H\&E (40X) Abundant lymphocytes along with few epitheloid cells.

The statistical analysis to compare pleural effusion $(n=71)$ of CS and CB for cellularity-mild, moderate and marked by chi square test showed statistical significance $(p<0.05)$. Comparison of malignant effusion done with McNemar's test for $\mathrm{CB}$ $\left(x^{2}=12.02\right)$ showed that $C S$ method was not superior to $\mathrm{CB}$ method in pleural fluid analysis.

The statistical analysis to compare peritoneal fluid ( $n=43$ ) of CS and CB for cellularity- mild, moderate and marked by chi square test showed statistical significance $(\mathrm{p}<0.05)$. Comparison of malignant effusion done with McNemar's test for CB $\left(\mathrm{x}^{2}=8.03\right)$ showed that CS method was not superior to $\mathrm{CB}$ method in peritoneal fluid analysis.

The stastistical analysis of malignant effusions $(\mathrm{n}=114)$ for cell block method for sensitivity is $100 \%$ (95\% CI: $54.05 \%$ to $100 \%$ ), Specificity $79.63 \%$ (95\% CI: $70.80 \%$ to $86.77 \%$ ), positive predictive value $21.43 \%$ (95\% CI: $8.35 \%$ to $40.96 \%$ ) and negative predictive value $100 \%$ (95\% CI: $95.76 \%$ to $100 \%$ ).

The total comparison $(n=114)$ of malignant serous effusions done with McNemar's test for CB $\left(x^{2}=21.01\right)$ showed that CS method was not superior to CB method.

The test provides strong evidence to reject the null hypothesis that the CB method was not superior to CS Method in serous effusions.

\section{DISCUSSION}

Cytological examination of serous effusions is of paramount importance in diagnostic, therapeutic and prognostic implications. Reactive mesothelial cells, abundance of inflammatory cells and paucity of representative cells contribute to considerable difficulties in making conclusive diagnosis on conventional smears $[3,5,6]$. It is important not only in the diagnosis of malignant lesions but also helps in staging and prognosis of the disease. Malignant cells in the pleural or the ascitic fluids were almost always indicative of metastatic tumours, as the primary malignancies which arose from the mesothelial cell lining were rare[7]. The development of malignant effusion is a common complication of pulmonary and gastric carcinomas .Examination of effusions also helps in detection of unknown primary [3].

The difficulty in diagnosis by CS method is either secondary to marked atypia of mesothelial cells caused by the microbiological, chemical, physical, immunological, or metabolic insults to the serous membranes or to the subtle cytomorphological features of some malignant neoplasms, particularly welldifferentiated adenocarcinomas. The problem may become compounded by artefacts from poor fixation, preparation, or staining techniques. Although the preparation of CS is a much simpler procedure than that of paraffin sections, it has limitations such as lack of tissue architecture. In some cases, appreciation of tissue architecture make diagnosis easier[8]. Another limitation of the conventional cytological examination of effusions is that it has a sensitivity of only $40-70 \%$ for the presence of malignant disease due to overcrowding of cells, cell loss and different laboratory processing methods. Others like reactive mesothelial cells, abundance of inflammatory cells and paucity of representative cells contribute to considerable difficulties in making conclusive diagnosis on conventional smears[2].

Since the introduction of the CB technique by Bahrenburg nearly a century ago, it has been used routinely for processing fluids. In 1928, Zemansky concluded that the CB method was superior to the CS technique and that examination of materials other than pleural and ascitic fluids were unreliable. Cancer cells in the pleural or ascitic fluid are almost always indicative of metastatic cancer, as tumours arising from mesothelial cells lining these spaces are rare. When present, the tumour cells are usually numerous and frequently clusters may be found. The glandular forms are more reliable on $\mathrm{CB}$. The demonstration of mucin in the tumour cells is evidence that they originate from a glandular epithelium[9]. Diagnostic problems arise whenever there is only marginal morphological distinction, for example, between reactive mesothelial cells and poorly differentiated malignant cells[10]. Earlier methods of CB preparations did not receive much attention, probably due to the lack of standardized technique. In fact the main problem with the $\mathrm{CB}$ preparation is the risk of losing material during preparation. Some researchers used agar, plasma/thromboplastin to bind the sedimented cells, but they have some disadvantages[8].

Hence, in this study an attempt was made to prepare and analyse both smears and cellblock from the 
same specimen. Due consideration was given to age, sex, site of effusion, clinical and radiological findings to arrive at final diagnosis and also to identify primary malignant lesion.

In this study, $10 \%$ alcohol-formalin was used as a fixative for cellblock preparation to get better cellularity when compared to conventional smears as formalin minimized the cell loss by forming protein cross links and gel formation as done by other workers[3]. Cell blocks prepared from residual tissue fluid can be used as adjuncts to smear for establishing a more definitive cytopathological diagnosis.

We received 114 samples of body cavity fluids of which pleural fluid samples contributed more i.e. $62 \%$. Peritoneal fluid samples were $38 \%$ as is the case with Sears et al. [7].

The age range included in our study was from 16 to 88 years. Male to Female ratio was 1.53:1. Majority of women were in $5^{\text {th }}$ decade while two peaks were seen in $4^{\text {th }}$ and $6^{\text {th }}$ decades. The least number of samples were in the paediatric age (4 cases). In other studies $[5,11]$ the maximum number of cases among women were in $4^{\text {th }}$ and in $7^{\text {th }}$ decade among men. The binomial age distribution in males was because of maximum number of tuberculosis cases in the age group of 31-40 years.

Cellblock study revealed increased cellularity, better morphological details due to preservation of architectural patterns like three dimensional clusters, better nuclear and cytoplasmic preservation and intact cell membrane. Maximum number of singly scattered cells were seen in CS method whereas glands and sheets were seen in $\mathrm{CB}$ method.Similar findings were noted in various studies[2, 3, 6,9].

In the present study of 114 cases on CS Method, 52 cases (45\%) had mild cellularity, 40 cases (35\%) moderate cellularity and marked cellularity in 22 cases $(20 \%)$ whereas in cell block method 12 cases (10\%) had mild cellularity, 54 cases $(50 \%)$ had moderate cellularity and 45 cases $(40 \%)$ had marked cellularity. Similar findings were also seen in Udasimath et al. [12].

Pseudoacinar or acinar structures and nucleoli were better appreciated in our study when compared to conventional smears. The reactive or atypical mesothelial cells which simulate malignancy in smears were identified as reactive or mesothelial cells by cellblock method. Similar findings were noticed in Dekker and Bupp[4] study.

In the present study a significant increase in diagnostic yield for malignancy by cellblock method. Additional 19\% (22 cases) malignant lesions were identified by cellblock method in comparison to conventional smear study. In addition $11 \%$ (12 cases) of the suspicious cases in conventional smear were diagnosed as malignant lesion by cellblock method.

In study done by Bodele et al. [3], additional $7 \%$ (10 cases) of malignant lesions were identified by cellblock method. Dekkar and Bupp[4] study, reported that samples obtained by combined cellblock method and smear technique for malignant lesions were double to that of conventional smear technique only. By using cellblock method tumours were subsequently demonstrated in $38 \%$ of the patient who had negative or atypical cytological reports.

In a study done by Khan et al. [13] additional findings were diagnostic in $16 \%$ of malignant cases. Additional 18 cases for malignant lesions were diagnosed by cellblock method in study done by Takagi $\mathrm{F}[14]$.

Khan et al. [5], in another study titled as usefulness of cellblock verses smears in malignant effusion cases reported that the recovery rate for malignant lesions by cellblock preparation was $20 \%$ greater than that obtained for specimen examined in smear only.

According to various studies additional diagnostic yield for malignancy was noted if conventional smear technique is supplemented by cellblock method[3, 6, 15].

In our study, by using cellblock method we diagnosed malignant lesions in $19 \%$ of samples, where as in conventional smear method diagnosis for malignant lesion was $5 \%$ only. Most of the tumours were of adenocarcinoma type.

In the present study besides $\mathrm{CS}$ and $\mathrm{CB}$ methods we confirmed the benign and malignant cases by history, clinical examination, radiological examination, FNAC and histopathological examination wherever necessary.

We noted the presence of pericellular lacunae in more than $50 \%$ cases of adenocarcinoma, especially of mucin secreting type, characterized by large cell clusters. Price et al. [16] study also has revealed the presence of pericelular lacunae especially in cases of adenocarcinoma samples.

The morphological evaluation of cytological specimens from body cavity fluids faces difficulties in the differential diagnosis between benign reactive mesothelial cells and adenocarcinoma or malignant mesothelioma. So, different antibodies serve as reliable markers in the differential diagnosis of reactive mesothelial cells, adenocarcinona and malignant mesothelioma in serous effusions This diagnostic dilemma can be furthered settled by staining with PAS 
stain, D-PAS, Alcian blue and Hyaluronidase blue. Introduction of $\mathrm{CK} 5 / 6$ and calretinin helps in differentiating mesothelioma from adenocarcinoma[17], [18].

The cell block technique has an added advantage that multiple sections of the same material can be obtained for special stains and immunohistochemistry[5]. Apart from this advantage, morphological details can also be obtained with the cell block method, which include preservation of the architectural pattern like cell balls and papillae and three dimensional clusters, excellent nuclear and cytoplasmic details, and individual cell characteristics. On the other hand, fragments of tissue can easily be interpreted in a biopsy-like fashion. It is thus concluded from the present study that it is advisable to study paraffin sections by using the cell block method before discarding specimens that are negative for malignant cells by smear examination.

\section{CONCLUSION}

In present study, statistical analysis revealed highly significant difference between conventional smear and cellblock methods. Cellblock technique by using $10 \%$ alcohol - formalin as a fixative is simple, inexpensive and does not require any special training or instrument. Morphological features were better identified by cellblock method, when compared to conventional smear method. Multiple sections can be obtained if required for special stain or IHC study. Sensitivity for malignant lesion by cellblock method was significantly increased as compared to conventional smear. CB method is superior to CS method.

\section{REFERENCES}

1. Spriggs, A. I., \& Boddington, M. M. (2016). The cytology of effusions: pleural, pericardial and peritoneal and of cerebrospinal fluid. ButterworthHeinemann.

2. Shivakumarswamy, U., Arakeri, S. U., Karigowdar, M. H., \& Yelikar, B. R. (2012). Diagnostic utility of the cell block method versus the conventional smear study in pleural fluid cytology. Journal of Cytology/Indian Academy of Cytologists, 29(1), 11.

3. Grandhi, B., Shanthi, V., Rao, N. M., Reddy, V. C., \& Mohan, K. (2014). The diagnostic utility of cell block as an adjunct to cytological smears. International Journal of Medical Research \& Health Sciences, 3(2), 278-284.

4. Dekker, A., \& Bupp, P. A. (1978). Cytology of serous effusions: An investigation into the usefulness of cell blocks versus smears. American journal of clinical pathology, 70(6), 855-860.

5. Khan, N., Sherwani, K. R., Afroz, N., \& Kapoor, S. (2006).Usefulness of cell blocks versus smears in malignant effusion cases, J. Cytol., 23(3),129.
6. Sujathan, K., Kannan, S., Mathew, A., Pillai, K. R., Chandralekha, B., \& Nair, M. K. (2000). Cytodiagnosis of serous effusions: A combined approach to morphological features in papanicolaou and may-grunwald giemsa stained smears and a modified cell block technique, J. Cytol. 17(2),89.

7. Khan, N., Sherwani, K. R., Afroz, N., \& Kapoor, S. (2006). Usefulness of Cell Blocks Versus smears in Malignant effusion cases. Journal of cytology, 23(3), 129.

8. Kung, I. T., Yuen, R. W., \& Chan, J. K. (1989). Optimal formalin fixation and processing schedule of cell blocks from fine needle aspirates. Pathology, 21(2), 143-145.

9. Vellios, F., \& Griffin, J. (1954). Examination of body fluids for tumor cells. American journal of clinical pathology, 24(6), 676-681.

10. Bousfield, L. R., Greenberg, M. L., \& Pacey, F. (1985). Cytogenetic diagnosis of cancer from body fluids. Acta cytologica, 29(5), 768-774.

11. Nathan, N. A., Narayan, E., Smith, M. M., \& Horn, M. J. (2000). Cell block cytology: improved preparation and its efficacy in diagnostic cytology. American journal of clinical pathology, 114(4), 599-606.

12. Udasimath, S., Arakeril, S. U., Karigowdar, M. H., \& Yelikar, B. R. (2012). The Role of the Cell Block Method in the Diagnosis of Malignant Ascitic Fluid Effusions. Journal of Clinical \& Diagnostic Research, 6(7).

13. Khan, N., Sherwani, R. K., Afroz, N., \& Kapoor, S. (2005). Cytodiagnosis of malignant effusion and determination of primary site. Journal of cytology, 22(3), 107.

14. Takagi, F. (1954). Studies on tumor cells in serous effusion. American Journal of Clinical Pathology, 24(6), 663-675.

15. Güldaval, F., Anar, C., Polat, G., Gayaf, M., Yavuz, M. Y., Korkmaz, A., \& Aydoğdu, Z. (2019). Contribution of cell block obtained by thoracentesis in the diagnosis of malignant pleural effusion. Journal of cytology, 36(4), 205.

16. Dey, S., Nag, D., Nandi, A., \& Bandyopadhyay, R. (2017). Utility of cell block to detect malignancy in fluid cytology: Adjunct or necessity?. Journal of Cancer Research and Therapeutics, 13(3), 425.

17. Hyun, T. S., Barnes, M., \& Tabatabai, Z. L. (2012). The diagnostic utility of D2-40, calretinin, CK5/6, desmin and MOC-31 in the differentiation of mesothelioma from adenocarcinoma in pleural effusion cytology. Acta cytologica, 56(5), 527 532.

18. Subbarayan, D., Bhattacharya, J., Rani, P., Khuraijam, B., \& Jain, S. (2019). Use of panel of markers in serous effusion to distinguish reactive mesothelial cells from adenocarcinoma. Journal of cytology, 36(1), 28. 